\title{
Fast Computation Algorithm for Discrete Resonances among Gravity Waves
}

\author{
Elena Kartashova \\ RISC, J. Kepler University, Linz, Austria \\ E-mail: lena@risc.uni-linz.ac.at
}

Traditionally resonant interactions among short waves, with large real wavenumbers, were described statistically and only a small domain in spectral space with integer wave-numbers, discrete resonances, had to be studied separately in resonators. Numerical simulations of the last few years showed unambiguously the existence of some discrete effects in the short-waves part of the wave spectrum. Newly presented model of laminated turbulence explains theoretically appearance of these effects thus putting a novel problem - construction of fast algorithms for computation of solutions of resonance conditions with integer wave-numbers of order $10^{3}$ and more. Example of such an algorithm for 4-waves interactions of gravity waves is given. Its generalization on the different types of waves is briefly discussed.

PACS numbers: 47.10.-g, 47.27.De, 47.27.T, 02.60.Pn

\section{INTRODUCTION}

Classical theory of wave turbulence was developed for description of statistic properties of weakly nonlinear waves in infinite domains. Importance of resonant interactions among these waves was first pointed out in 1961 by O.Phillips ${ }^{1}$ and this led to construction ${ }^{2}$ of the first kinetic equation. Later on it was established by many researchers that effects of finite length are not described by the classical theory and kinetic equations, and 20 years later, in 1981, O.Phillips wrote that "new physics, new mathematics and new intuition is required" 3 in order to understand energetic behavior of these systems. Discrete effects appear when waves "notice" boundary conditions, i.e. wave lengths are comparable to the sizes of interaction domain, and therefore wave numbers are integers. First theoretical result on the behavior 


\section{Elena Kartashova}

of discrete waves systems was published in $1990 \mathrm{in}^{4}$ where main distinction between continuous and discrete wave systems was established: stochastic interactions between all waves (infinite domain) versus interactions within small independent groups of waves and existence of non-interacting waves (finite domain). Theory of discrete wave systems was then presented in ${ }^{5}$ thus giving a rise to the following qualitative picture of wave turbulence: short waves are described by power energy spectra and kinetic equations, long waves are described by Clipping method and dynamic equations. Numerous papers of last few years showed that this qualitative picture has to be modified because some discrete effects (frozen turbulence, mesoscopic turbulence, etc.) are also observable ${ }^{7}$ in the short-waves' part of the wave spectra. Discovery of intermittent patterns in that part of wave spectra which is supposed to be described by a kinetic equation only, gave us the reason to revisit the very foundations of wave turbulence theory. It turned out that its basic theoretical ground - KAM theorem - is proven not for all short waves, a countable number of short waves with rationally connected frequencies is excluded from consideration leaving some "gaps" in the wave spectrum. These gaps correspond to the waves whose ratio of frequencies is algebraic number of degree $\leq 2$, for instance, a rational number which is usually understood as necessity for the corresponding dispersion function to be a rational function. A model of laminated turbulence was recently presented ${ }^{9}$ which consists of two layers, discrete one (in the whole range of wave numbers) and continuous one (in the short-waves' part of the wave spectrum). The main fact allowing to construct this model is following: frequencies of discrete resonantly interacting waves are rationally connected though the dispersion function itself can be highly irrational. Thus, the model of laminated wave turbulence "fills" the gaps left by KAM theory and explains, in particular, coexistence of power energy spectra and coherent structures in the short-waves' part of wave spectrum.

Model of laminated turbulence brings our attention to a completely novel question: how to compute discrete resonances in some large computation domain? Indeed, say for 4-waves interactions of 2-dimensional gravity waves, the resonance conditions can be regarded in the form

$$
\sqrt{k_{1}}+\sqrt{k_{2}}=\sqrt{k_{3}}+\sqrt{k_{4}}, \quad \vec{k}_{1}+\vec{k}_{2}=\vec{k}_{3}+\vec{k}_{4},
$$

where $\vec{k}_{i}=\left(m_{i}, n_{i}\right), \forall i=1,2,3,4$ and $k_{i}=\left|\vec{k}_{i}\right|=\sqrt{m_{i}^{2}+n_{i}^{2}}$. It means that in a finite but big enough domain of wave numbers, say $|m|,|n| \leq 1000$, direct approach leads to necessity to perform computations with integers of order $10^{6}$. These computations in a substantially smaller domain $|m|,|n| \leq 128$ took 3 days ${ }^{10}$ with Pentium-4. 


\section{Discrete Resonances among Gravity Waves}

The main goal of this paper is to show that constructions of independent classes of resonantly interacting waves ${ }^{6}$ can be re-formulated as a pure mathematical procedure which allows to reduce drastically computation time for this sort of equations. Gravity waves are taken as our main example. Some necessary results on discrete resonances of gravity waves are given in ${ }^{6}$ but formulations there are too formal and partly inaccurate. We reformulate all necessary results on construction of classes for these waves in Sec.2 and give some illustrative examples. In Sec. 3 we describe the computation algorithm and some preliminary results of numerical simulation. Brief discussion is given in Sec.4.

\section{CONSTRUCTION of CLASSES}

In this section we regard 4-waves resonances of 2-dimensional gravity waves in the form

$$
\begin{aligned}
\sqrt{k_{1}}+\sqrt{k_{2}} & =\sqrt{k_{3}}+\sqrt{k_{4}} \\
\vec{k}_{1}+\vec{k}_{2} & =\vec{k}_{3}+\vec{k}_{4} .
\end{aligned}
$$

To construct independent classes of resonantly interacting we need following definition.

\section{Definition}

Let $\vec{k}=(m, n)$ be a vector with integer coordinates, $m, n \in \mathbb{Z}$. Represent the square root of the norm $|\vec{k}|$ as

$$
\left(m^{2}+n^{2}\right)^{1 / 4}=\gamma q^{1 / 4}, \quad \gamma, q \in \mathbb{N}
$$

and $q$ does not contain fourth degrees:

$$
q=p_{1}^{\alpha_{1}} \cdots p_{s}^{\alpha_{s}}, \quad \alpha_{i} \in\{1,2,3\}
$$

with all different prime $p_{i}$. Number $q$ is called index of a vector $\vec{k}=(m, n)$ and number $\gamma$ is called its weight. Class $C l_{q}$ of index $q$ is set of all vectors with this index.

For instance, vectors $(1,3)$ and $(12,4)$ belong to the same class $C l_{10}$ of index 10 , with weights $\gamma=1$ and $\gamma=2$ correspondingly, while vector $(2,1)$ lies in $\mathrm{Cl}_{5}$ of index 5, with weight $\gamma=1$.

\section{Lemma}

Let vectors $\vec{k}_{1}, \vec{k}_{2}, \vec{k}_{3}, \vec{k}_{4}$ construct a solution of (1), then only two cases are possible: 


\section{Elena Kartashova}

Case 1: all vectors belong to the same class,

Case 2: all vectors belong to two different classes $C l_{q_{1}}, C l_{q_{2}}$ in such a way that there exist $q_{1}, q_{2}$ :

$$
\vec{k}_{1}, \vec{k}_{3} \in C l_{q_{1}} \quad \text { and } \quad \vec{k}_{2}, \vec{k}_{4} \in C l_{q_{2}}
$$

or

$$
\vec{k}_{1}, \vec{k}_{4} \in C l_{q_{1}} \quad \text { and } \quad \vec{k}_{2}, \vec{k}_{3} \in C l_{q_{2}} .
$$

In the Case 2 all solutions are symmetric, i.e. vectors belonging to each class must have the same norm. All asymmetric solutions, if any, are described by the Case 1 .

The statement of Lemma follows immediately from elementary properties of integers and we are not going to the detailed proof here. General idea of the proof is very simple indeed: two different irrational numbers can not satisfy any equation with rational coefficients. For instance, equation $a \sqrt{3}+b \sqrt{5}=c$ has no solutions for arbitrary rational $a, b, c$. It means that irrationalities corresponding to classes indices in (1) have to be rid off in order to construct its integer solutions. In general, one to four different irrationalities can appear in (1), simple consideration show that all cases but two described below give no integer solutions.

In the Case 1, all four irrationalities are equal and can be canceled, so that (1) is reduced to

$$
\gamma_{1} q^{1 / 4}+\gamma_{2} q^{1 / 4}=\gamma_{3} q^{1 / 4}+\gamma_{4} q^{1 / 4} \Rightarrow \gamma_{1}+\gamma_{2}=\gamma_{3}+\gamma_{4} .
$$

Let us regard as example a couple of asymmetric solutions given in ${ }^{8}$ :

$$
(-4,0),(49,0),(9,0),(36,0) \text { and }(-20,15),(-20,-15),(-49,0),(9,0) \text {. }
$$

An easy check shows that all 8 vectors belong to the same class $C l_{1}$ of index 1 , with weights $\gamma_{1}=2, \gamma_{2}=7, \gamma_{3}=3, \gamma_{4}=6$ and $\gamma_{1}=5, \gamma_{2}=5, \gamma_{3}=$ $7, \gamma_{4}=3$ correspondingly, so that condition $\gamma_{1}+\gamma_{2}=\gamma_{3}+\gamma_{4}$ is full-filled in both cases. It is important to understand that 4 vectors constructing some asymmetric solution must belong to the same class but not necessarily to $C l_{1}$ as in the solutions above. In the next section some asymmetric solutions are presented which lie in other classes.

In the Case 2, the irrationalities with corresponding coefficients in front of them must be pair-wise equal to be canceled, for instance

$$
\gamma_{1} q_{1}^{1 / 4}+\gamma_{2} q_{2}^{1 / 4}=\gamma_{3} q_{1}^{1 / 4}+\gamma_{4} q_{2}^{1 / 4} \Rightarrow \gamma_{1}=\gamma_{3} \quad \text { and } \quad \gamma_{2}=\gamma_{4} .
$$




\section{Discrete Resonances among Gravity Waves}

Notice that Lemma gives only necessary condition of the existence of a solution. This means that to find a solution we have first to construct classes, then find solutions within the corresponding classes and check linear conditions afterwards. Obviously, (1),(2) have infinitely many solutions - for instance, quartets

$$
\vec{k}_{1}=(a, b), \quad \vec{k}_{2}=(c, d), \quad \vec{k}_{3}=(a, b), \quad \vec{k}_{4}=(c, d)
$$

and

$$
\vec{k}_{1}=(a, b), \quad \vec{k}_{2}=(c, a-b+c), \quad \vec{k}_{3}=(b, a), \quad \vec{k}_{4}=(a-b+c, c)
$$

with arbitrary integers $a, b, c, d$ give its solution, as well as any proportional quartets corresponding to multiplication of all wave-numbers on the same integer. Less trivial example of "tridents"

$$
\vec{k}_{1}=(a, 0), \quad \vec{k}_{2}=(-b, 0), \quad \vec{k}_{3}=(c, d), \quad \vec{k}_{4}=(c,-d)
$$

possess two-parametric series of solutions ${ }^{8}$ :

$$
a=\left(s^{2}+t^{2}+s t\right)^{2}, b=\left(s^{2}+t^{2}-s t\right)^{2}, c=2 s t\left(s^{2}+t^{2}\right), d=s^{4}-t^{4}
$$

with arbitrary integer $s, t$. This series gives solutions of both (1),(2) though perhaps not all of them. Parametrization (6) is constructed in such a way that norms of all four vectors are full squares, i.e. again vectors $\vec{k}_{1}, \vec{k}_{2}, \vec{k}_{3}, \vec{k}_{4}$ belong to the same class $C l_{1}$ of index 1 , with weights

$$
\gamma_{1}=s^{2}+t^{2}+s t, \quad \gamma_{2}=s^{2}+t^{2}-s t, \quad \gamma_{3}=\gamma_{4}=s^{2}+t^{2},
$$

providing $\gamma_{1}+\gamma_{2}=\gamma_{3}+\gamma_{4}$ for any parameters $s, t$.

There is no known way to construct general solution of (1),(2) and even a construction of some particular solutions' series demands a lot of skillful work and a bit of luck. On the other hand, this hard work is mostly not well-paid because coming back to physical problem setting, we usually need all solutions in some spectral domain, not only those which could be nicely parameterized. In the next section we use Lemma to construct fast computer algorithm for finding solutions of (1),(2) which is a challenging numerical problem.

\section{SCHEME of NUMERICAL ALGORITHM}

As it was shown in previous section, equations (1),(2) have infinitely many solutions. But not all of them are interesting from physical point of 


\section{Elena Kartashova}

view. We follow ${ }^{11}$ in classification (written out up to the cyclic change of indices) of all possible solutions according to their role in energy transfer : (I) trivial resonances $\vec{k}_{1}=\vec{k}_{3}, \vec{k}_{2}=\vec{k}_{4}$ which do not redistribute the energy, an example is given by (3); (II) symmetrical resonances $\left|\vec{k}_{1}\right|=\left|\vec{k}_{3}\right|,\left|\vec{k}_{2}\right|=\left|\vec{k}_{4}\right|$ which do not generate new wavelengths and therefore do not transfer the energy through the scales, an example is given by (4); (III) asymmetrical resonances playing major role in energy transfer, an example is given by (5). In this paper we are primary interested in asymmetric solutions which means in terms of Lemma that all vectors belong to the same class (Case 1). Not going into all programming details we present here just underlying main ideas of our algorithm and first results of computer simulations.

Step 1. Create first auxiliary array of primes $A_{p}$ in the given spectral domain $p \leq D$ using Eratosthenes' Sieve procedure which eliminates composite numbers from the list of natural numbers and can be briefly described as follows:

- Create an array $A_{d}$ containing all integers $1, \ldots, d$, write number "1" into array $A_{p}$, mark "1" in the array $A_{d}$ as used element;

- Introduce cycle variable $i=1, \ldots, \sqrt{d}$;

- Find first number in $A_{d}$ which is greater than 1 and is not marked yet as a composite, denote it as $r$ and mark all numbers $2 r, 3 r, 4 r, \ldots$ as composite (at the first step $r=2$, at the second $r=3$ and so on);

- write number $r$ into array of primes $A_{p}$;

- put $i=r$ and repeat the procedure.

Step 2. Create second auxiliary array $A_{q}$ of possible indexes constructed of the primes obtained at the Step 1 . Indexes are computed directly by formula

$$
q=p_{1}^{\alpha_{1}} \cdots p_{s}^{\alpha_{s}}, \quad \alpha_{i} \in\{1,2,3\},
$$

in the domain $q \leq 2^{1 / 4} \sqrt{d}<1.19 \sqrt{d}$. Say, for $d=1000$, it is enough to compute for $q \leq 37$.

Step 3. Find all integer solutions of the linear equation

$$
\gamma_{1}+\gamma_{2}=\gamma_{3}+\gamma_{4}
$$

in the domain $\gamma_{i} \leq 2^{1 / 4} \sqrt{d}, \quad \forall i=1, . .4$. 


\section{Discrete Resonances among Gravity Waves}

Remark. Further it will be necessary to check when expressions under the radicals $\gamma^{4} q$ can be represented as a sum of two squares. Here one has to make use of the well-known Euler theorem: representation of a positive number as a sum of two squares which is possible iff each of its prime factors of the form $4 t+3$ occurs as an even power. For instance, pair $\gamma=3, q=1$ has 3 in even degree, expression under the integral is $3^{4} \cdot 1=81$, representation as a sum of two squares exists and number $81=9^{2}+0^{2}$ should be written into the array $A_{\gamma, q}$. On the other hand, for pair $\gamma=2, q=3$, the expression under the radical has factor 3 in the odd power, i.e. number 48 can not be represented as a sum of two squares and it is not an element of the array $A_{\gamma, q}$. Notice that all prime factors of $\gamma$ have even powers which means that only prime factors of $q$ has to be investigated. Consequently, an arbitrary presentation of $q$ as a sum of two squares, multiplied by $\gamma^{4}$, gives corresponding presentation of the expression under the radical.

Step 5. Check Euler theorem for all elements of $A_{q}$ and construct array $\tilde{A}_{q}$ with "allowed" elements only; find all presentations as a sum of two squares for each "allowed" element. Obviously, one number can be decomposed into sum of two squares in more then one way, for instance $1105=4^{2}+33^{2}=9^{2}+32^{2}=12^{2}+31^{2}=23^{2}+24^{2}$.

Remark. Due to Lemma, the search of 2-square-representations for the numbers $\leq 2 d^{2}$ is reduced to the numbers $<1.19 \sqrt{d}$. In particular, for $d=1000$ we have to compute these presentations for the numbers in the domain $\leq 37$ which can be done by direct enumeration. The number $S$ of different 2-square-presentations of an integer is proportional to the difference between its prime factors of the form $4 t+1$ and $4 t+3$ (Lagrange theorem).

Step 6. For each $q \in \tilde{A}_{q}$ and each its possible 2-square-presentation, $q=$ $\tilde{m}_{j}^{2}+\tilde{n}_{j}^{2}, j=1,2, \ldots, S$, compute all under-integral-expressions $\gamma_{i}^{4}\left(\tilde{m}_{j}^{2}+\tilde{n}_{j}^{2}\right)$ and check linear conditions on $m_{j}, n_{j}$ :

$$
\begin{gathered}
m_{1}+m_{2}=m_{3}+m_{4}, \\
n_{1}+n_{2}=n_{3}+n_{4},
\end{gathered}
$$

with $m_{1}=\gamma_{1}^{2} \tilde{m}_{j}, n_{1}=\gamma_{1}^{2} \tilde{n}_{j}, \quad m_{2}=\gamma_{2}^{2} \tilde{m}_{j}, n_{2}=\gamma_{2}^{2} \tilde{n}_{j}, \quad m_{3}=\gamma_{3}^{2} \tilde{m}_{j}, n_{3}=$ $\gamma_{3}^{2} \tilde{n}_{j}, m_{4}=\gamma_{4}^{2} \tilde{m}_{j}, n_{4}=\gamma_{4}^{2} \tilde{n}_{j}$.

Summarizing, Lemma allows to reduce domain of integers under consideration from $2 d^{2}$ to $2^{1 / 4} \sqrt{d}$ while classical procedure (Eratosthenes' Sieve) and some known number-theoretical results (Euler and Lagrange theorems) allow to further reduce the computation time which is about $4 \mathrm{~min}$. at 


\section{Elena Kartashova}

Pentium-4 for spectral domain $m_{i}, n_{i} \leq 1000$. In this domain we have found five asymmetric solutions which are not tridents:

$$
\begin{aligned}
& \vec{k}_{1}=(495,90), \vec{k}_{2}=(64,128), \vec{k}_{3}=(359,118), \vec{k}_{4}=(200,100), \\
& \vec{k}_{1}=(675,225), \vec{k}_{2}=(64,192), \vec{k}_{3}=(479,237), \vec{k}_{4}=(260,180), \\
& \vec{k}_{1}=(810,45), \vec{k}_{2}=(128,192), \vec{k}_{3}=(598,117), \vec{k}_{4}=(340,120), \\
& \vec{k}_{1}=(855,360), \vec{k}_{2}=(64,256), \vec{k}_{3}=(599,356), \vec{k}_{4}=(320,260), \\
& \vec{k}_{1}=(990,180), \vec{k}_{2}=(128,256), \vec{k}_{3}=(718,236), \vec{k}_{4}=(400,200) .
\end{aligned}
$$

These solutions belong to classes $\mathrm{Cl}_{5}, \mathrm{Cl}_{10}, \mathrm{Cl}_{13}, \mathrm{Cl}_{17}, \mathrm{Cl}_{20}$ correspondingly, with weights $\gamma_{1}=15, \gamma_{2}=8, \gamma_{3}=13, \gamma_{4}=10$ for all solutions.

\section{Brief discussion}

We described here first version of our algorithm in order to show how to use Lemma for reducing drastically computation time. At present, this algorithm is written only for positive integer wave numbers $m_{i}, n_{i} \in \mathbb{N}$. Using some simple symmetrical considerations, one can adapt it for the case of arbitrary $m_{i}, n_{i} \in \mathbb{Z}$. Another simple modification can be made in order to find solutions for the case

$$
\sqrt{k_{1}}=\sqrt{k_{2}}+\sqrt{k_{3}}+\sqrt{k_{4}}, \quad \vec{k}_{1}=\vec{k}_{2}+\vec{k}_{3}+\vec{k}_{4}
$$

or for the case of 5 -wave interactions and more (of course, Lemma should be re-formulated).

Moreover, basing on this algorithm, one can develop generic algorithm for fast computation of discrete resonances among different types of waves with dispersion function $\omega$ being an arbitrary polynomial function of the norm of wave vector, $\omega=\omega(k)$. Different dispersion functions in case of two dimensional waves will have different formula for index (Step 2 of present algorithm); in case of three dimensional waves, i.e. $k=|\vec{k}|=\sqrt{m^{2}+n^{2}+l^{2}}$, Lagrange and Euler theorems should be replaced by some known numbertheoretical results on the decomposition of an integer into the sum of three squares. All other algorithmic steps will be the same as above. Development of such a generic algorithm for a big class of dispersion functions is our current object of interest. 


\section{Discrete Resonances among Gravity Waves}

\section{ACKNOWLEDGMENTS}

Author is very grateful to Sergey Nazarenko for valuable discussions and remarks. Author acknowledges support of the Austrian Science Foundation (FWF) under projects SFB F013/F1304.

\section{REFERENCES}

1. O. Phillips Fluid Mech. 9, 193 (1960)

2. K. Hasselman Fluid Mech. 12, 481 (1962)

3. O. Phillips Fluid Mech. 106, 215 (1981)

4. E.A. Kartashova. Physica D, 46, 43 (1990)

5. E.A. Kartashova. Phys. Rev. Let., 72, 2013 (1994); E.A. Kartashova. AMS Transl. 182 (2), 95 (1998); E.A. Kartashova. Theor. Math. Phys. 99, 675 (1994)

6. E.A. Kartashova. AMS Transl. 182 (2), 95 (1998)

7. See for instance: A.N. Pushkarev, V.E. Zakharov. Physica D 135, 98 (2000); V.E. Zakharov, A.O. Korotkevich, A.N. Pushkarev, A.I. Dyachenko. JETP Letters 82 (8), 487 (2005); Y.V. Lvov, S. Nazarenko, B. Pokorni. Eprint arXiv:math-ph/0507054 v3 (2006)

8. Y.V. Lvov, S. Nazarenko, B. Pokorni. Eprint arXiv:math-ph/0507054 v3 (2006)

9. E.A. Kartashova. JETP Letters 83 (7), 341 (2006)

10. S. Nazarenko. Private communication (12.2005)

11. B. Pokorni. PhD Thesis, Rensselaer Polytechnical Institute, Troy, New York (2005) 